\title{
Evaluating the benefits of paste fill using advanced schedule optimisation techniques
}

\author{
B.M. Maybee MIRARCO - Mining Innovation, Laurentian University, Canada \\ S. Hall MIRARCO - Mining Innovation, Laurentian University, Canada \\ A. Tousignant MIRARCO - Mining Innovation, Laurentian University, Canada
}

\begin{abstract}
Operational decisions within the underground mining industry have the ability to offer substantial increases in value if they can be made early in the project planning stage. The choice of fill technology is one of these operational decisions that have the potential to add significant value to the project. However, due to the manually intensive requirements to schedule the operations under multiple designs to allow for the comparison of the different technologies, the benefits are usually undervalued.

In many cases, the benefits from a change in fill technology are limited to an evaluation of the sensitivity of the design to changes based on a modification in fill rate and curing times. What is generally not taken into account, due to time constraints, is that a change in fill technology may also allow for introduction of a completely new mining sequence. This might require a different equipment fleet and a completely different schedule, which may dramatically enhance the value of the project. These are not mutually exclusive decisions, and if a proper evaluation of the fill technology is to be undertaken, then all of the potential benefits and pitfalls must be included as the competing scenarios are evaluated for potential implementation.

The Schedule optimisation tool (SOT) developed at MIRARCO uses advanced technology and computing power to identify and evaluate thousands of sequences and scheduling alternatives in a relatively short period of time. Through its optimisation process, SOT uses a powerful genetic algorithm to create multiple sequences of increasing value as it completely re-orders the activity set to find better sequences and schedules for the given design. Alternate designs can be considered in the same manner and new research is underway to develop rapid evaluation of multiple design options. As a result, a full scenario evaluation is possible, accounting for the fact that an improved mining sequence and equipment fleet utilisation may exist for a given change in fill technology.

This paper describes how SOT can be used to identify the true scheduling benefits (as measured by net present value) that can be realised by a mining project through the use of paste fill. Through the use of case study data, it is demonstrated that the value of the mine can be substantially increased if all of the decision variables, both timing and operational efficiencies, related to that choice are accounted for in the evaluation.
\end{abstract}

\section{Introduction}

Operational decisions within the underground mining industry have the ability to offer substantial increases in value if they can be made early in the project planning process. The choice of fill material is one of these operational decisions. Due to the manually intensive requirements to schedule the operations under multiple designs, the benefits are usually undervalued as only a handful of sequencing and scheduling alternatives are evaluated. In many cases, the quantification of the benefits that arise from a change in fill material is limited to an evaluation of the sensitivity of a design to modifications in the fill rate and curing times. What is not taken into account is that a change in fill technology may also allow for the introduction of a completely new mining sequence, requiring a different equipment fleet and a completely different schedule, which may dramatically enhance the value of the project. These are not mutually exclusive decisions, and if a proper evaluation is to be undertaken, all of the potential sequencing alternatives must be included in the analysis. 
This paper describes the SOT that has been developed at MIRARCO for use in underground mine scheduling. Through the use of SOT, thousands of feasible mine schedules under various strategies can be evaluated, providing helpful information to decision-makers in the planning process. A case study is presented to illustrate how this advanced computing power and automation can be used to add important information early in the decision-making process so that an informed decision about the fill material to be used can be made. In this case study, the scheduling benefits that can be realised by a mining project will be identified for the assumptions made, using the maximisation of net present value (NPV) as the optimisation objective.

\section{$2 \quad$ Mine design and scheduling}

Optimisation is the process of maximising or minimising a function. In the context of underground mine scheduling, optimisation usually involves maximising the NPV of the project. Many attempts have been made using various optimisation techniques to find this optimised value. However, these techniques tend to fall short of finding an optimum due to the huge search space of underground mine scheduling alternatives.

The optimal solution of scheduling problems is a topic of interest to many in the operations research field, with the solution methods being categorised as either exact or heuristic. Exact optimisation strategies can be described as 'brute force' methods that iterate through all of the potential solutions (Poniewierski - Runge, 2003, pers. comm.). However, Blattman (2003) noted that when the search space is immense, optimising with any exact technique quickly becomes infeasible. Using simulation tools for scheduling in a surface mine environment, Pegman et al. (1996) found it necessary to limit the search space.

Heuristic methods use experience as a means of narrowing the search space (rules of thumb, educated guesses, common sense, etc.), and have been introduced to optimise underground mine scheduling due to the immense size and complexity of the problem. One form of heuristic solution to mine scheduling has been the use of rules. However, these methods are pre-determined in many cases, and the solution provided is merely what the process dictated (Poniewierski - Runge, 2003, pers. comm.). More advanced solutions are found using heuristic searches rather than heuristic rules. One of the simplest heuristic search techniques is hill climbing, which evaluates alternatives in the neighbourhood of the current solution for their ability to increase value. Its main drawback is the number of cases that need to be evaluated as the number of decision parameters increases (Hall, 2003).

Alternatively, a linear programming problem involves a finite number of continuous decision variables, and can be solved by simple methods that are quite efficient at finding a solution. There are two models that comprise the scheduling problem - the sequencing model and the multi-period planning model. The sequencing model addresses the problem of scheduling a finite number of activities by relating the activities to one another through dependencies, as one activity will depend upon the initiation or completion of another. The multi-period planning model addresses the problem of resource allocation over time. In this model, there are a finite number of time periods of equal length, and the decisions about resource inputs are made at all these periods, with the set of acceptable decisions at each period being defined by resource constraints.

Unfortunately, these models suffer when applied to underground mine scheduling due to their inability to handle large numbers of integer variables. In underground mining, schedules are created for discrete mining activities (development drive, stope, etc.). These activities need to be scheduled as complete units and cannot be broken into smaller pieces to satisfy the requirements of a linear programming model.

Finally, genetic algorithms (GAs) have been identified as one of the best techniques currently available for getting close to an optimum where there is no analytical solution (Hall and Stewart, 2004). A GA can be viewed as a generalisation of the hill climbing technique. It addresses the problem of climbing to a local optimum by simultaneously considering a number of solutions in different 'neighbourhoods' of the search space, and has been successfully applied to the underground mine scheduling problem in combination with heuristic rules as a precursor to SOT, which is described in the next section (Fava Lindon et al., 2005). Although this does not guarantee arriving at the global optimum, it typically improves upon a simple model by at least 10 to $15 \%$ (Michalewicz, 1992). 


\subsection{Schedule optimisation tool (SOT)}

SOT has been developed at MIRARCO through applied research projects with industry partners. It is a planning tool that allows mine planners to quickly identify feasible and high-quality mine schedules. In its optimisation process, SOT uses a powerful GA to search thousands of feasible scheduling alternatives to identify those that maximise the NPV of the project. It creates these schedules by completely re-ordering the activities that are to be sequenced (development, stopes, backfill, etc.) as it evolves towards 'better' scheduling alternatives for the given design, and this process has been demonstrated through 'real world' applications (Maybee et al., 2009). Alternate designs can be considered in the same manner and, as a result, a full scenario evaluation is possible, accounting for the fact that an improved mining sequence and equipment fleet utilisation may exist for a given change in technology.

The main features of SOT that contribute to NPV improvements include the application of constraints, justin-time development, heuristic guidance and learning. Figure 1 illustrates the optimisation process flow that is used. SOT adheres to all operational constraints that have been established, including precedence constraints and resource capacity constraints. SOT produces smoothed schedules, i.e. schedules adhering to all identified capacity constraints on activity properties expressed as tonnages or lengths. For example, when an ore tonnage cap (resource capacity constraint) is used, not only does SOT ensure that this limit is never exceeded, but it also strives to achieve the specified level as closely as possible. In an attempt to identify better schedules, SOT then performs a sliding process, in which costly development activities are pushed off to just-in-time without breaking caps or required scheduling lags. This process not only has the potential to defer costs, but also boost revenues by reallocating the resources. The result is an optimised schedule that adheres to multiple scheduling constraints.

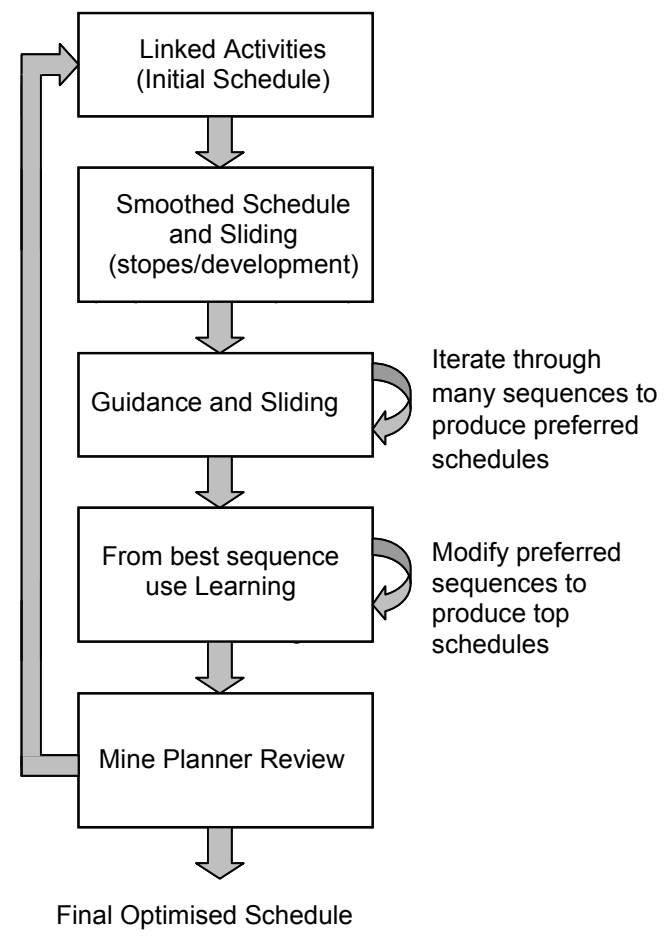

\section{Figure 1 SOT process}

The search for improved mine schedules can begin from a random set of schedules generated by SOT. However, because the search space is so large, this may lead to an unnecessarily long run time. Thus, heuristic guidance of the GA was incorporated into SOT. A set of guidance formulas is provided to rank the mining activities using attributes that are currently used by mine planners (i.e. rank the stoping activities by highest grade). This ranking is then used to prioritise the scheduling of both the stoping and required development activities. This approach was found to provide a good initial starting point for the optimisation, effectively narrowing the search space to be investigated and directing the search towards schedules with higher NPVs, resulting in a much reduced search time. Heuristic guidance of the planning process potentially 
sacrifices the globally optimum NPV, but directs the search to areas where it can quickly find high quality schedules based on a simple ranking scheme.

SOT repeatedly produces a set of valid schedules and then combines them to 'learn' better schedules. The learning (by means of the GA) uses a selection and crossover process to build on the schedules developed through constraint adherence, guidance and sliding of activities. Through this process, the best scheduling alternatives are selected and combined to form new alternatives, constructing progressively more optimal schedules. While the many features within SOT allow it to create improved scheduling options, there are also many ways in which users can manipulate the tool to provide answers to their questions.

Figure 2 illustrates three ways of using SOT, distinguished by the degree of manual scheduling performed by the mine planner before SOT is employed. First, the mine planner can produce the set of activities with appropriate linking, and provide to SOT an initial schedule, having made no attempt to adhere to constraints. The ordering of activities in the initial schedule is interpreted by SOT as specifying the relative priorities of the activities. SOT will smooth out the schedule so that no resource or sequencing constraints are broken. Profitable activities will be pushed off if necessary to correct the breaking of a cap. At the same time, the smoothed schedule pulls forward other profitable activities to closely follow the ore cap where possible, and slides off development until it is needed. Second, the mine planner can provide some scheduling (an intermediate schedule), perhaps with some constraints being broken. With even more effort, the mine planner can produce a final schedule as input to SOT. In each case, the ordering of activities in the mine planner's schedule is interpreted by SOT as a ranking of the activities.

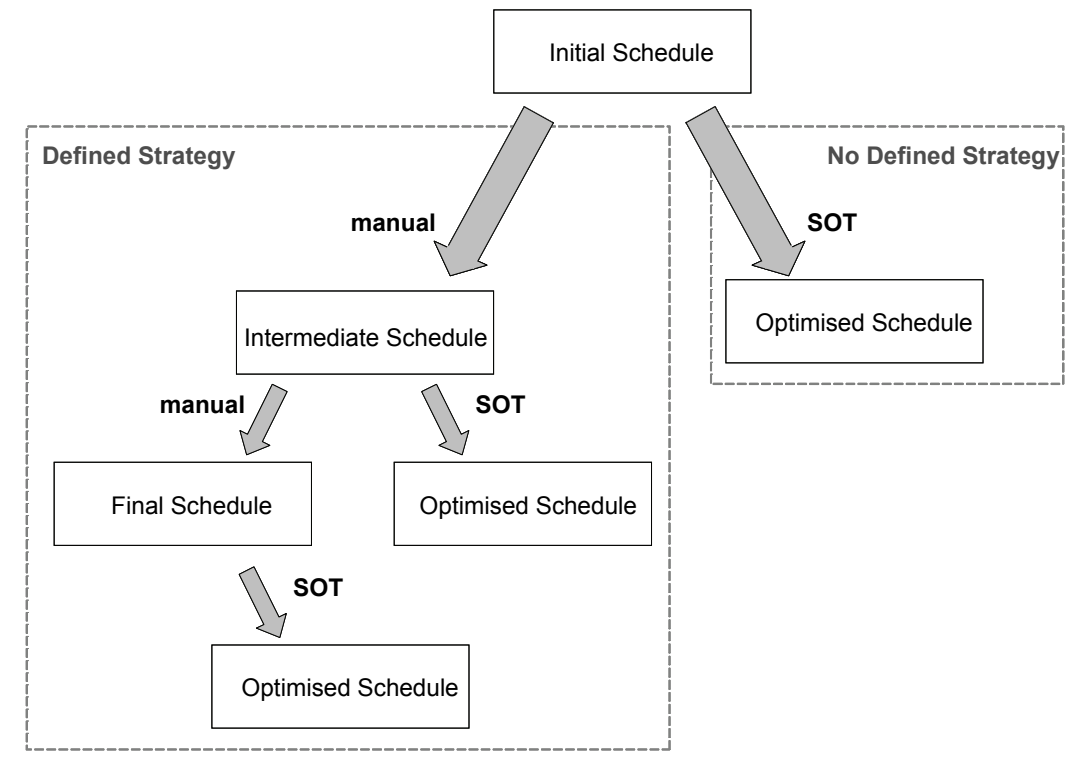

\section{Figure 2 Different approaches to using SOT}

\section{Case study}

This section of the paper presents the application of SOT to a hypothetical underground mining project. The purpose of this case study is to show that, by using the advanced computational power of SOT, many alternative sequencing and scheduling options for this mining project can be investigated in a relatively short period of time. Also, this process can be undertaken very early in the planning process when a significant impact on the value of the project can be made.

Amaratunga and Yaschyshyn (1997) described the three main types of backfill as hydraulic fill, cemented rock fill and paste fill. Most hydraulic fills contain coarse tailings in a slurry form that can be easily delivered to the fill location through boreholes. As a result, hydraulic fill can be pumped into place at a relatively high rate. However, as it has a large water content, more curing time is required for adequate drainage, as well as increased level preparation and clean-up times.

Cemented rock fills are placed mechanically, usually containing coarse aggregate waste rock from the operations. Through the addition of a cement-filled slurry, a very strong bond is created, making this type of 
fill very attractive in high-stressed areas. However, as the aggregate is not placed in a slurry form, filling a stope is a very time consuming process, and longer curing times for the cement slurry are still required.

Paste fill usually contains both fine and coarse tailings and is delivered to the fill location in a dense slurry form. Amaratunga and Yaschyshyn (1997) describe the benefits of paste fill as follows:

- strengths approaching rock fill can be achieved, while using less cement than hydraulic fills

- facilitates a rapid mining sequence because strength is achieved earlier compared with hydraulic fill

- allows the use of waste rock and slags as well as the fine fraction of tailings, thereby reducing surface tailings impoundment requirements

- paste fill has higher stiffness than hydraulic sand fill because of reduced porosity

- decant water from the fill is virtually eliminated, reducing costs and problems associated with barricade set-up/level clean-up and wear on mine dewatering pumps

- the borehole delivery system of slurry fills can be used.

To investigate the benefits of these different fill materials, a hypothetical narrow vein gold mine has been created containing 102 stopes accessed by a single decline in the middle of the operations. As a result, the mine is divided into an east and a west ore zone that share the decline for both access and haulage of materials to the surface. From this design, the mine will extract a total of 331,607 tonnes of ore at an average grade of 9.6 grams per tonne, producing $2,900 \mathrm{~kg}$ of gold. The mine will need to extract 135,659 tonnes of waste material to develop the stoping areas within this design. The layout shown in Figure 3 requires that no underground voids are left after the operations have completed. As a result, the physical design requires 197,531 tonnes of backfill be used to fill the mined out stopes.

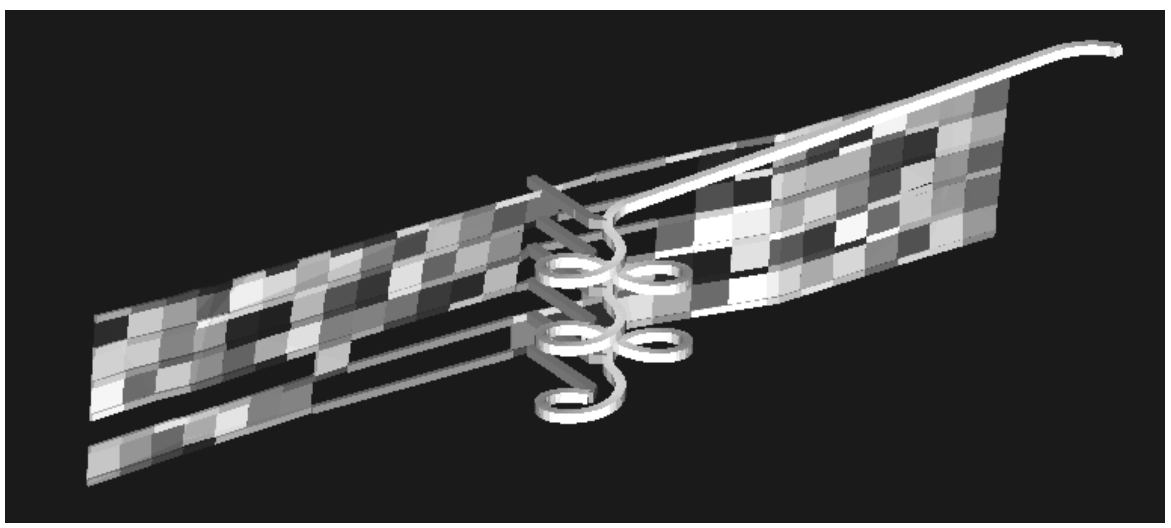

\section{Figure 3 Mine layout}

For SOT to be used as the scheduling tool for this mining project, it requires that the design be divided into separate mining activities (stoping, development and backfill). Each of these activities will have an associated tonnage, length and mineral grade that will be used in both the scheduling and evaluation processes. Also, since the activities are to be scheduled, mining rates and predecessor relationships are required. Table 1 shows the mining rates that will be used for the different activity types in this mining project.

It is also recognised that some of the resources used in the mines extraction will be constrained. For example, there are a specific number of development crews available within the mine. For this case study, it is being assumed that one development crew is available. Also, due to the haulage considerations, only two stopes can be producing at any given time. Likewise, due to constraints on the backfill plant, only two stoping voids can be filled at any time. 
Table 1 Mining rates

\begin{tabular}{ll}
\hline Activity Type & Rate \\
\hline Decline & 2 metres/day \\
Level access & 2 metres/day \\
Level waste development & 3 metres/day \\
Stope undercut & 3 metres/day \\
Stoping & 350 tonnes/day \\
\hline
\end{tabular}

Finally, to perform a financial evaluation based on NPV, certain corporate parameters are required. These parameters identify information such as the cost of extracting the different mining activities, the price that can be recognised for the extracted mineral in the market, and the corporate discount rate. Table 2 shows the corporate parameters that are being assumed for this case study.

Table 2 Corporate scheduling parameters

\begin{tabular}{ll}
\hline Parameter & Amount \\
\hline Gold price & $\$ 1,000 /$ ounce \\
Development cost & $\$ 5,000 /$ metre \\
Stoping cost & $\$ 100 /$ tonne \\
Backfill cost & $\$ 50 /$ fill tonne \\
Discount rate & $10 \%$ \\
\hline
\end{tabular}

\subsection{Methodology}

This case study evaluates the scheduling benefits that can be achieved from moving from one fill material to another. As outlined in Amaratunga and Yaschyshyn (1997), there are three main types of backfill; hydraulic fill, rock fill, and paste fill. To assess the benefits of using these different materials within the hypothetical narrow vein long hole mine, three scenarios are evaluated using a typical primary-secondary mining sequence. Within this sequence, primary and secondary stopes are identified in the design. The sequence then must mine and backfill both neighbouring primaries before a secondary stope can be extracted, as highlighted by the circled area in Figure 4.

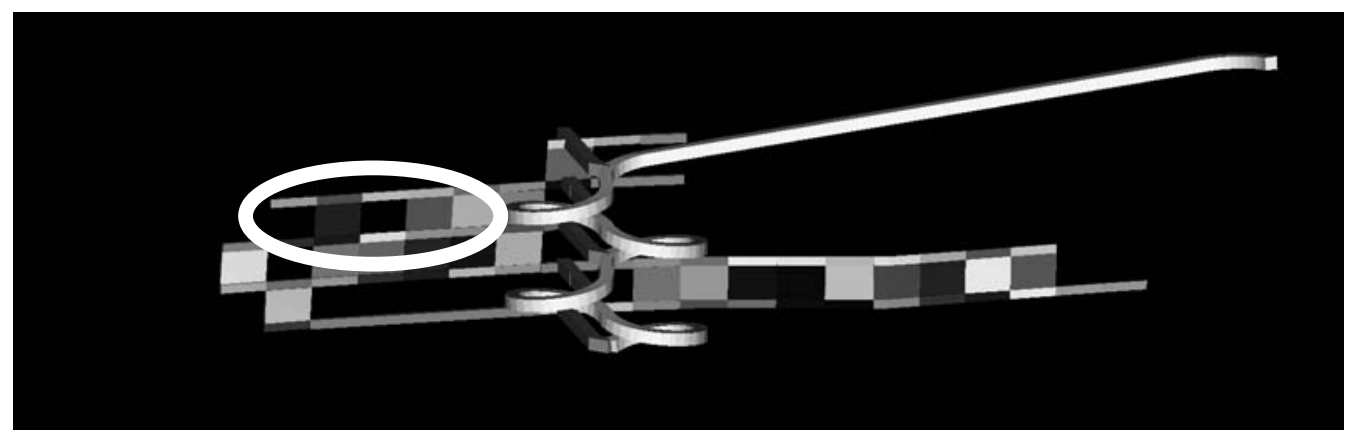

\section{Figure 4 Pre-defined primary-secondary mining sequence}

Each of these scenarios can be run very quickly (producing about 5,000 scheduling options within a 10 minute time span for this hypothetical mining operation). To start, SOT will be used to evaluate the mine using each type of fill material, with the only differences being the rate at which the voids can be filled, and the curing time required before the neighbouring stope can be extracted. Table 3 shows the figures for backfill rate and curing time used in this case study. 
Table 3 Fill parameters

\begin{tabular}{llll}
\hline Scenario & Backfill Type & Fill Rate & Curing Time \\
\hline 1 & Hydraulic fill & 750 tonnes/day & 10 days \\
2 & Rock fill & 350 tonnes/day & 8 days \\
3 & Paste fill & 1,500 tonnes/day & 5 days \\
\hline
\end{tabular}

After comparing the results of these three scenarios, a fourth scenario is developed for the paste fill option. Due to the increased strength that can be achieved with paste fill, it is assumed that the strict primary-secondary design rule can be relaxed as highlighted by the circled area in Figure 5. In this case, two adjacent stopes are mined before their neighbours on either side due to the strength of the paste fill being used.

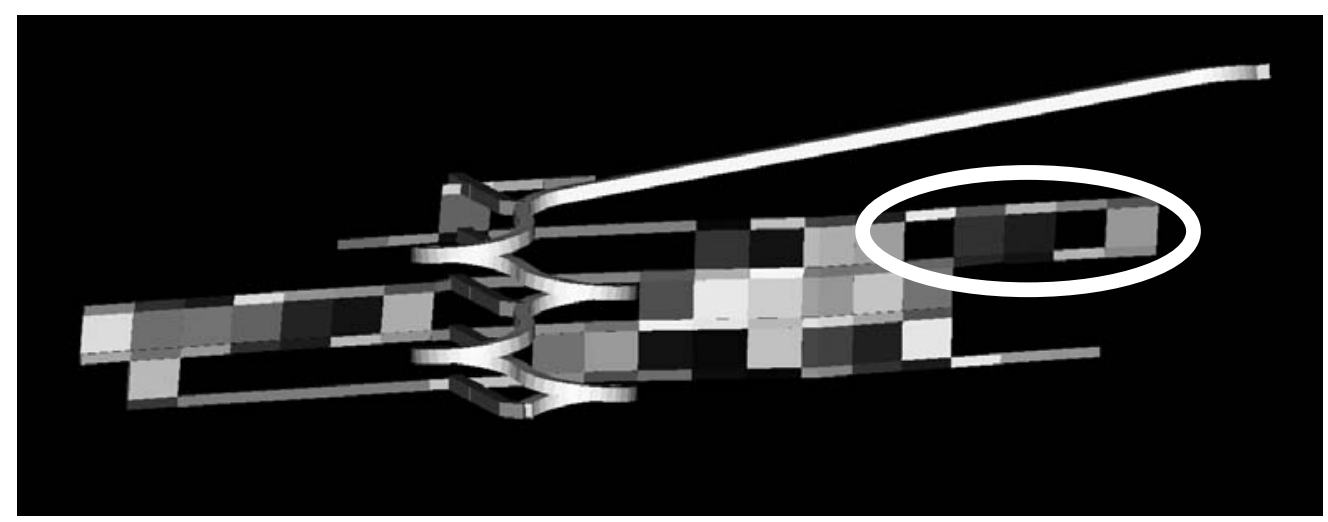

Figure 5 Primary-secondary sequence selected by SOT

In this scenario, the same constrained resources and parameters are maintained as in scenarios 1 to 3 , but now the stopes designed as secondaries can be taken before their neighbouring primaries if it increases the value. In this fashion SOT is allowed to decide what stopes should be primaries and what should be secondaries instead of pre-defining these labels. This assumption takes advantage of the optimisation and automated scheduling power provided by SOT to identify the full sequencing benefits that paste fill can offer.

\subsection{Results}

This section shows the results of the analysis described in section 3.1. Using SOT, schedules for the narrow vein long hole mine are optimised under the assumptions of Scenarios 1 to 3. For each scenario, SOT investigated more than 10,000 scheduling options, with the results presented in Table 4. It is readily apparent that a small increment in the maximum value from one scenario to the next has been recorded by only adjusting the fill rates and cure times. It should be noted that the standard deviations in Table 4 are in respect to the discrete scheduling options for each scenario, and do not apply to a single schedule. As a result, the small difference in value that is recorded from one scenario to another is a true difference. Although this is a highly constrained and relatively small mining project, it is obvious that, with the implementation of paste fill in Scenario 3, the average, maximum and minimum NPVs are all increased over Scenarios 1 and 2. It is also noticed that the standard deviation of the scheduling results is dramatically decreased, as the use of paste fill offers more potential high-value options. This becomes an important factor when it is recognised that unforeseen events will occur in an underground mining environment, and having more high-value options allows for flexibility within the mining project. 
Table 4 Results for Scenarios 1, 2 and 3

\begin{tabular}{lllll}
\hline Scenario & Maximum NPV & Average NPV & Minimum NPV & Standard Deviation \\
\hline 1 & $\$ 33,042,519$ & $\$ 32,698,267$ & $\$ 30,871,072$ & $\$ 229,916$ \\
2 & $\$ 33,072,042$ & $\$ 32,707,038$ & $\$ 31,002,180$ & $\$ 236,835$ \\
3 & $\$ 33,116,138$ & $\$ 32,792,732$ & $\$ 31,795,656$ & $\$ 137,610$ \\
\hline
\end{tabular}

SOT was again used to evaluate scheduling options for Scenario 4, where the primary-secondary rule was relaxed, with the results presented in Table 5.

Table 5 Results for Scenarios 3 and 4

\begin{tabular}{lllll}
\hline Scenario & Maximum NPV & Average NPV & Minimum NPV & Standard Deviation \\
\hline 3 & $\$ 33,116,138$ & $\$ 32,792,732$ & $\$ 31,795,656$ & $\$ 137,610$ \\
4 & $\$ 33,216,893$ & $\$ 33,147,959$ & $\$ 32,898,270$ & $\$ 94,002$ \\
\hline
\end{tabular}

In this analysis, SOT investigated more than 10,000 scheduling options in less than an hour of processing time, and it can be seen that a slight increase in the expected value of the project is obtained. However, what is important to note is that through the use of SOT, no additional set up is required to run this fourth scenario. Instead, a different curing time and rule set regarding selection of primary and secondary stopes are entered, followed by pressing the 'run button' to set SOT off on its investigation. Likewise, this analysis could continue to test the effects of even quicker fill rates and curing times by simply adjusting a few settings.

\section{Conclusion}

The planning of underground mines requires a significant amount of time in the preparation and smoothing of activity based schedules. This paper describes a SOT that automates a portion of the underground mine planning process so that additional focus can be on other value-adding tasks. Time that would otherwise be needed for scheduling can instead be invested in, for example, a complete evaluation of the benefits (and threats) from the implementation of alternative fill materials.

Through the use of a case study, it has been shown that four independent scenarios, related to the fill material used and sequencing constraints, can be thoroughly investigated in a relatively short period of time. In this analysis, more than 100,000 scheduling alternatives were identified and evaluated across the four scenarios in less than 48 hours, creating more data for the decision-making process, and fostering the ability to ask even more complex and technical questions than in the past. By incorporating advanced software tools and computing power within the underground mine planning process, the opportunity exists to create more optimal underground mine designs.

\section{References}

Amaratunga, L. and Yaschyshyn, D. (1997) Development of a high modulus paste fill using fine gold mill tailings, Geotechnical and Geological Engineering, Vol. 15, pp. 205-219.

Blattman, M. (2003) Creation of an underground mine development sequencing optimizer, In Proceedings 2003 SME Annual Meeting, Cincinnati, USA, Preprint 03-064, 8 p.

Fava Lindon, L., Goforth, D., van Wageningen, A., Dunn, P., Cameron, C. and Muldowney, D. (2005) A parallel composite genetic algorithm for mine scheduling, In Proceedings 9th IASTED International Conference on Artificial Intelligence and Soft Computing, A.P. del Pobil (ed), Anaheim: ACTA Press, pp. 245-250.

Hall, B. (2003) How mining companies improve share price by destroying shareholder value, presented at CIM Mining Conference and Exhibition, Montreal, www.amcconsultants.com.au, 31 p.

Hall, B. and Stewart, C. (2004) Optimising the Strategic Mine Plan - Methodologies, Findings, Successes and Failures, Orebody Modelling and Strategic Mine Planning Symposium: Spectrum Series 14, Orebody Modelling and Strategic Mine Planning: Uncertainty and Risk Management, R. Dimitrakopoulos (ed), Perth: AusIMM, pp. 281-288. 
Maybee, B., Fava, L., Dunn, P., Wilson, S. and Fitzgerald, J. (2009) Towards optimum value in underground mine scheduling, presented at CIM AGM, Toronto, USA, $10 \mathrm{p}$.

Michalewicz, Z. (1992) Artificial Intelligence, Berlin, Germany, Springer-Verlag.

Pegman, M., Forward, N., King, B. and Teal, D. (1996) Mine Planning and Scheduling at RTZ Technical Services, Customer Technical Papers, www.ilog.com/products/optimization/tech/custpapers.cfm, 10 p. 
